\title{
Value of computed tomography angiographic collateral status in prediction of malignant middle cerebral artery infarction
}

Sasitorn Petcharunpaisan, M.D. ${ }^{(1)}$

Wannaporn Ngernbumrung, M.D. ${ }^{(1)}$

Sukalaya Lerdlum, M.D. ${ }^{(2)}$

From ${ }^{(1)}$ Department of Radiology, King Chulalongkorn Memorial Hospital,

The Thai Red Cross Society, Bangkok, Thailand.

${ }^{(2)}$ Department of Radiology, Faculty of Medicine, Chulalongkorn University, Bangkok, Thailand.

Address correspondence to S.P. (e-mail: sasitorn.pe@chulahospital.org)

\section{Abstract}

Objective: To determine whether the collateral status evaluated by single phase computed tomographic angiography (CTA) helped prediction of Malignant middle cerebral artery infarction (mMCAi) in patients with large arterial occlusion who did not receive endovascular treatment.

Materials and Methods: We retrospectively reviewed patients with acute is chemic stroke in anterior circulation in our institute from January 2015 to December 2015. We analyzed clinical data, baseline National Institutes of Health Stroke Scale (NIHSS), Alberta Stroke Program Early CT Score (ASPECTS) on baseline nonenhanced computed tomography of the brain (NECT brain), and CTA collateral status. " mMCAi" was defined based on clinical criteria.

Results: Thirty-five patients were included. The mean age was $68.8 \pm 15.56$ years. The mean baseline NIHSS and baseline ASPECTS were $17( \pm 5)$ and $6( \pm 3)$, respectively. All patients received intravenous thrombolysis. CTA collateral status and baseline NECT ASPECTS were significantly correlated with development of mMCAi (P-value $=0.007$ and 0.001$)$. Only baseline NECT ASPECTS was an 
independent predictive factor for mMCAi (OR 0.63, 95\%CI 0.46-0.86, P-value $=0.004)$. Patients with baseline NECT ASPECTS $\leq 7$ were more likely to develop mMCAi (OR 14.29 95\%CI 1.57-129.94, P-value 0.018).

Conclusion: In times of acute stroke, patients with proximal MCA or ICA occlusion received intravenous thrombolysis alone, baseline NECT ASPECTS and CTA collateral status were significantly correlated with development of mMCAi. However, only baseline ASPECTS $\leq 7$ was an independent predictor for mMCAi.

Keywords: CTA collateral status, Malignant MCA infarction, NECT ASPECTS, Acute ischemic stroke.

\section{Introduction}

Stroke is one of the leading causes of death and disability worldwide. Large cerebral infarction due to occlusion of middle cerebral artery (MCA) can be a life-threatening condition. Acute infarction involving a large portion of cerebral hemisphere with associated space-occupying cerebral edema, leading to rapid neurological deterioration has been termed "malignant MCA infarction (mMCAi)" $[1,2]$. Brain edema with a subsequent midline shift, brain herniation and increased intracranial pressure (ICP) may lead to coma and death. Significant space occupying effect usually manifests between the second and the fourth days after stroke onset; however, it possibly occurs more rapidly within 24 hours $[1,2]$. Up to eighty percent of patients with mMCAi treated with conservative medical therapy alone developed coma and death within 2 to 5 days. Surviving patients were left with severe disability and poor quality of life. Early treatment with decompressive surgery (hemicraniectomy) could reduce ICP, extensive brain edema and further infarction when performed within 48 hours after stroke onset [3]. Decompressive craniectomy not only reduces mortality, but also improves functional outcome and reduces longstanding critical care therapy. Thus, it is important to identify predictors of mMCAi which may help in management planning [3-6]. 
Cerebral collateral circulation is necessary to maintain cerebral blood flow and penumbra when cerebral arterial insufficiency due to thromboembolism, hemodynamic disturbance or a combination occurs $[7,8]$. Baseline collateral status is correlated with baseline infarct volume and infarct growth over 24 hours [9]. Recent studies demonstrated effect modification by collateral status on relationship between recanalization and good clinical outcome $[9,10]$. Collaterals impact efficacy and safety of endovascular treatment in acute ischemic stroke patients; patients with more robust collateral circulation had better recanalization, reperfusion and functional outcomes, as well as lower periprocedural symptomatic intracranial hemorrhage and mortality [10-16]. In these recent years, assessment of collateral circulation with multiphase CTA showed advantages over single phase CTA giving information on the degree and extent of pial arterial filling in a time resolved manner and has been used in The Endovascular Treatment for Small Core and Anterior Circulation Proximal Occlusion with Emphasis on Minimizing CT to Recanalization Times (ESCAPE) trial as a criterion for patient selection to endovascular treatment $[17,18]$.

Recent studies found that collateral circulation, assessment with both single phase and multiphase CTA, is one of the factors that independently predict malignant MCA infarction, together with baseline NIHSS, NECT ASPECTS and revascularization status $[4,7,19]$. Then we sought to determine whether CTA collateral status derived from single phase CTA may help prediction of mMCAi in acute stroke patients with large arterial occlusion who did not receive endovascular treatment. 


\section{Materials and methods}

\section{Patients}

We retrospectively reviewed patients with acute ischemic stroke in anterior circulation who underwent stroke fast tract imaging protocol in our institute from January 2015 to December 2015. Our stroke fast tract imaging protocol included NECT brain, CTA of the brain and neck and CT brain perfusion. The inclusion criteria were: 1) patient's age was 18 years old or above; 2) the onset of symptoms at the time performing fast tract image protocol was lower than 6 hours; 3 ) there was the presence of large arterial occlusion (internal carotid artery (ICA) or M1 segment of MCA) on CTA; 4) patient did not receive endovascular treatment; 5) patients may or may not receive intravenous administration of recombinant tissue-type plasminogen activator (rtPA). Exclusion criteria included: 1) the presence of intracranial hemorrhage on NECT; 2) the presence of previous large cerebral infarction in ipsilateral cerebral hemisphere or other intracranial space taking lesion on NECT; 3 ) poor image quality.

Patients' clinical information were collected from the hospital information system and outpatient records, including demographic data, clinical presentation, the onset of symptoms, onset-to-image time, NIHSS score, information of rtPA administration, and records of conscious deterioration and craniectomy during admission.

\section{CT imaging protocol}

All scans were obtained using 64 slice multidetector CT scanner installed at emergency department (Brilliance 64 Philips Health-care, Netherland). Parameters of NECT scan are as follows; a detector collimator of $64 \times 0.4 \mathrm{~mm}$, tube current of $300 \mathrm{~mA}$, tube voltage of $120 \mathrm{kV}$, and FOV of $25 \mathrm{~cm}$, covering from foramen magnum to the vertex. Axial image with 5-mm slice thickness was transferred to pictures achieving and information system (PACS). Parameters of CTA are as follows; detector collimation of $64 \times 0.6 \mathrm{~mm}$, tube current of $400 \mathrm{~mA}$, tube voltage of $120 \mathrm{kV}$, and field of view of $50 \mathrm{~cm}$, covering from aortic arch up to vertex. Acquisition was triggered using a bolus tracking $(100 \mathrm{HU})$ in the aortic arch after $80 \mathrm{~mL}$ of intravenous contrast medium (Ultravist ${ }^{\oplus} 300$ ) injection with 
an injection rate of $4 \mathrm{~mL} / \mathrm{s}$, followed by $40 \mathrm{~mL}$ saline flush. One-millimeter-thick axial maximal intensity projection (MIP) image, together with 8-millimeter-thick MIP image in axial, coronal and sagittal planes are transferred to PACS as routine image reconstruction protocol.

\section{Image analysis}

All CT images were reviewed by two readers (reader 1 was a second year neuroradiology fellow and reader 2 was a neuroradiologist with 8 -year experience in neuroimaging). The two readers were blind to clinical information, NECT and CTA results in each patient.

Baseline ASPECTS (Alberta Stroke Program Early CT Score) was evaluated on the initial axial 5-millimeter-thick NECT brain. CTA collateral status was evaluated on 8-millimeter-thick axial MIP images of CTA, using CTA collateral score derived from PROACT (Prolyse in Acute Cerebral Thromboembolism Trial) investigators with a scale of 0-3. According to this score, collaterals were defined as contrast filling pial arteries beyond the occluded site with different degrees on scale $0-3$ as follows; $0=$ absent collaterals supply to the occluded MCA territory; $1=$ collaterals filling $\leq 50 \%$ of the occluded territory; $2=$ collaterals filling $>50 \%$, but $<100 \%$ of the occluded territory; $3=$ collaterals filling $=100 \%$ of the occluded territory. Score $0-1$ defined poor collaterals and score 2-3 defined good collaterals.

"mMCAi" was defined when there were clinical records of decreased in the level of consciousness to give a score of 1 or more on item 1a of the NIHSS, according to ECASS (European Cooperative Acute Stroke Study) investigators [4, 5]. Hemorrhagic transformation was defined and graded as follows: HI type 1 $(\mathrm{HI}-1)=$ small heterogeneous petechiae along the margins of the infarct; HI type 2 $(\mathrm{HI}-2)=$ more confluent heterogeneous petechiae within the infarcted area; $\mathrm{PH}$ type $1(\mathrm{PH}-1)=$ homogeneous hematoma covering $\leq 30 \%$ of the infarcted area with mild space-occupying effect; $\mathrm{PH}$ type $2(\mathrm{PH}-2)=$ dense hematoma $>30 \%$ of the infarcted area with significant space-occupying effect. ( $\mathrm{HI}=$ hemorrhagic infarction, $\mathrm{PH}=$ parenchymal hemorrhage). Only $\mathrm{PH}-2$ was defined as symptomatic hemorrhagic transformation [20]. 


\section{Statistical analysis}

Patients were divided into mMCAi and non-mMCAi groups. Categorical variables were expressed in number (\%) and the continuous variable as the mean standard deviation. The 2 groups were compared for demographic categorical variables with the independent-sample T-test for continuous parameters and Chi-square test for categorical variables. Logistic regression was used to compute unadjusted and multivariable-adjusted odds ratios (OR) for the dichotomous outcomes (poor and good collateral status) of the mMCAi or non-mMCAi groups.

OR was presented with the associated $95 \%$ confidence intervals (CI). The threshold for statistical significance was set at $\mathrm{p}<0.05$. All statistical analyses were performed using the Statistical Package for the Social Sciences software version 16 (SPSS). Kappa statistics was made on the interrater agreement for CTA collateral score, baseline ASPECTS and symptomatic hemorrhagic transformation. The limits for Kappa statistics were the following; 0.4-0.6, moderate agreement; 0.6-0.8, good agreement; $>0.8$, excellent agreement.

\section{Results}

Thirty-five patients (19 women, 16 men; the mean age, 68.8 years \pm 15.56 ) fulilling all inclusion criteria, were selected. Twelve (34\%) patients developed mMCAi, whereas 23 patients (66\%) did not (non-mMCAi). All patients received intravenous rtPA therapy. Baseline characteristics of mMCAi and non-mMCAi patient groups were shown in Table 1 . 
Table 1: Baseline characteristics

\begin{tabular}{|c|c|c|c|c|}
\hline & $\begin{array}{c}\text { mMCAi } \\
(n=12,34 \%)\end{array}$ & $\begin{array}{l}\text { non-mMCAi } \\
(n=23,66 \%)\end{array}$ & Total & P-value \\
\hline Age, y, mean (SD) & $71.42( \pm 10.33)$ & $67.13( \pm 17.73)$ & $68.6( \pm 15.56)$ & 0.37 \\
\hline Female, $n, \%$ & $6(50 \%)$ & $13(57 \%)$ & $19(54 \%)$ & 0.73 \\
\hline \multicolumn{5}{|l|}{ Cardiovascular risk factors } \\
\hline $\mathrm{DM}, \mathbf{n}, \%$ & $2(17 \%)$ & $4(17 \%)$ & $6(17 \%)$ & 1 \\
\hline HT, n,\% & $4(33 \%)$ & $9(39 \%)$ & $13(37 \%)$ & 1 \\
\hline $\mathrm{AF}, \mathrm{n}, \%$ & $0(0 \%)$ & $2(9 \%)$ & $2(6 \%)$ & 0.53 \\
\hline DLD, $\mathbf{n}, \%$ & $2(17 \%)$ & $5(22 \%)$ & $7(20 \%)$ & 1 \\
\hline Onset, hours, mean (SD) & $2.84( \pm 1.3)$ & $2.95( \pm 1)$ & $2.91( \pm 1.1)$ & 0.8 \\
\hline $\begin{array}{l}\text { Baseline NIHSS score, mean } \\
\text { (SD) }\end{array}$ & $18( \pm 7)$ & $17( \pm 5)$ & $17( \pm 5)$ & 0.54 \\
\hline $\begin{array}{l}\text { Baseline ASPECTS, mean } \\
\text { (SD) }\end{array}$ & $4( \pm 2)$ & $7( \pm 2)$ & $6( \pm 3)$ & $0.001^{\star}$ \\
\hline Collateral status & & & & $0.007^{\star}$ \\
\hline Poor $(<2), n, \%$ & $12(100 \%)$ & $13(57 \%)$ & $25(71 \%)$ & \\
\hline $\operatorname{Good}(\geq 2), n, \%$ & 0 & $10(43 \%)$ & $10(29 \%)$ & \\
\hline
\end{tabular}

Note:- DM indicates diabetes mellitus; HT, hypertension; AF, atrial fibrillation; DLD, dyslipidemia; NIHSS, National Institutes of Health Stroke Scale; ASPECTS, Alberta Stroke Program Early CT Score; mMCAi, malignant middle cerebral artery infarction.

Only one patient developed symptomatic intracranial hemorrhage (PH-2). The mMCAi group had a significantly lower mean baseline ASPECTS compared to non-mMCAi group $4( \pm 2)$ versus $7( \pm 2)$, P-value $=0.001$. Collateral status was significantly correlated with development of mMCAi, P-value $=0.007$. None of patients with good collateral score developed mMCAi ( 0 versus 10$)$. On the other hand, other baseline clinical characteristics, including NIHSS, were not different among the two groups. 
In multivariate analysis, baseline ASPECTS was an independent predictive factor for mMCAi (OR 0.63, 95\%CI 0.46-0.86, P-value =0.004). Patients with baseline ASPECTS $\leq 7$ were more likely to develop mMCAi (OR 14.29 95\%CI 1.57-129.94, $\mathrm{P}$-value 0.018 ). On the other hand, collateral status evaluated by single phase CTA was not an independent predictor of mMCAi.

We did not find the correlation between clinical and imaging characteristics and development of symptomatic hemorrhagic transformation. (Table 2 and 3)

Table 2: Prediction of $m M C A i$ : multivariate binary regression analysis

\begin{tabular}{llll} 
& OR & $95 \% \mathrm{CI}$ & P-value \\
\hline Baseline NIHSS & 1.04 & $0.91-1.19$ & 0.53 \\
Baseline ASPECTS & 0.63 & $0.46-0.86$ & $0.004^{*}$ \\
Collateral status & 0.81 & $0.07-8.91$ & 0.86 \\
Baseline ASPECTS $\leq 7$ & 14.29 & $1.57-129.94$ & $0.018^{*}$
\end{tabular}

Note: CI, confidence interval; OR, odds ratio.

Table 3: Prediction of symptomatic hemorrhagic transformation ( $\mathrm{PH} 2)$ : multivariate binary regression analysis

$\begin{array}{llll} & \text { OR } & 95 \% \text { CI } & \text { P-value } \\ \text { Baseline NIHSS } & 1.03 & 0.69-1.53 & 0.87 \\ \text { Baseline ASPECTS } & 0.68 & 0.29-1.64 & 0.40 \\ \text { Collateral Status } & 0.43 & 0.01-19.4 & 0.66 \\ \text { Baseline ASPECTS } \leq 7 & 1.26 & 0.68-2.33 & 0.45\end{array}$

Note:- CI indicates confidence interval; OR, odds ratio. 
There was an excellent interrater agreement for CTA collateral grading (1.0). Interrater agreement for baseline NECT ASPECTS and hemorrhagic transformation were good (0.71) and excellent (0.86), respectively. Some examples of cases are shown in Figure 1 and Figure 2.

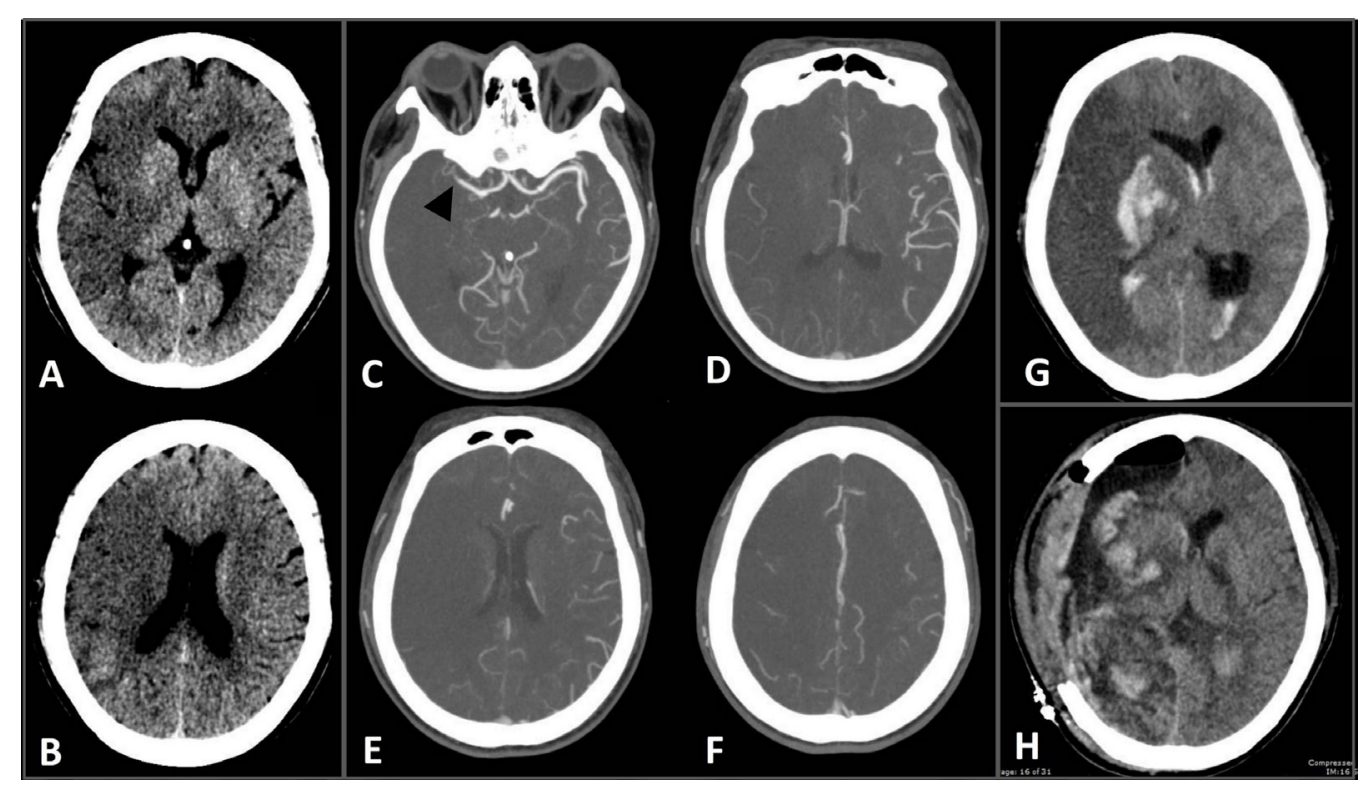

Figure 1. A 52-year-old female with acute right MCA infarction. Baseline NECT at 3 hours after onset ( $A$ and $B$ ) showed large hypodensity change in right cerebral hemisphere, ASPECTS = 1. Eight-mm axial MIP CTA (C-F) showed occlusion at right distal M1 (black arrowhead in $C)$ with poor collaterals, score $=1$; collaterals filling $\leq 50 \%$ of the occluded territory. Follow up NECT at 24 hours $(G)$ showed large edematous infarction with hemorrhagic transformation in right basal ganglia extending to lateral ventricles, midline shift and hydrocephalus. Decompressive craniectomy was done and follow up NECT at 24 hours (H) showed less degree of midline shift and improved hydrocephalus. New infarction in left globus pallidus was noted. 


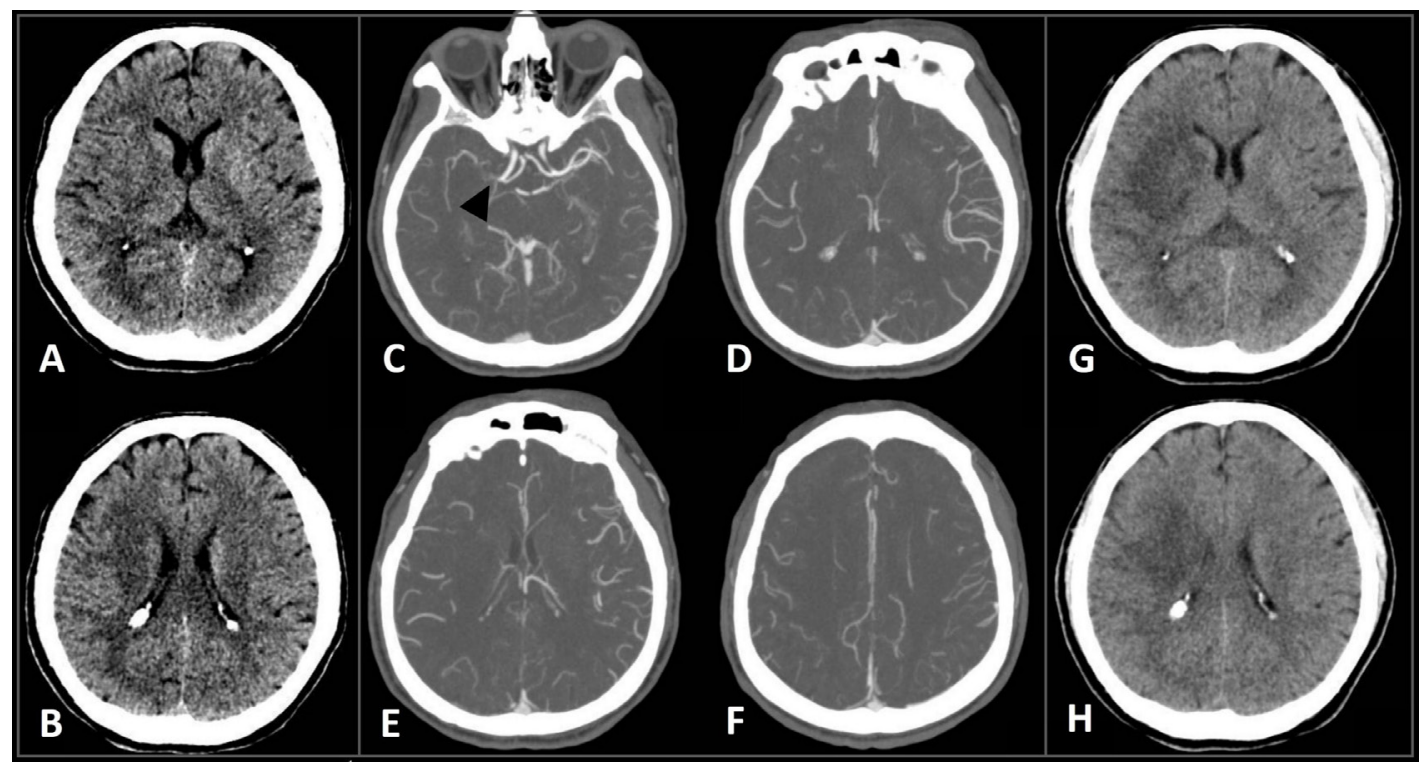

Figure 2. A 49-year-old male with acute right MCA infarction. Baseline NECT at 2 hours after onset $(A$ and $B)$ showed small hypodensity in posterior right insular cortex and right lentiform nucleus, ASPECTS $=8$. Eight-mm axial MIP CTA $(C-F)$ showed occlusion at right proximal M1 with good collaterals, score $=3$; collateral filling $=100 \%$ of the occlusion territory). Follow up NECT at 24 hours ( $G$ and $H$ ) showed small infarction in right insular lobe without hemorrhagic transformation.

\section{Discussion}

Malignant MCA infarction is a serious complication of acute ischemic stroke involving large area of MCA territory. Life-threatening brain edema usually develops within thefirst few days after the stroke onset and may cause a midline shift and brain herniation. Clinical worsening and fatal outcome is up to $80 \%$ in conservative case $[1,3,5]$. Early decompressive craniectomy may help improve the functional outcome and reduce mortality. Imaging may help early predict this condition and facilitate prompt surgical management $[3,6]$. 
In these recent years, studies assessing collateral status in acute ischemic stroke emphasize on the impact on clinical outcome. Noninvasive CTA collateral score grading pial arterial filling of contrast media beyond the occluded site has been extensively used to evaluate the status of collateral circulation in patients with acute stroke. Data from IMS III trials and systematic review and meta-analysis revealed that in patients receiving endovascular treatment; robust collateral status was correlated with smaller infarct volume, reduced further infarct growth, reduced periprocedural symptomatic intracranial hemorrhage, better clinical outcome at 3 months, and could be used to select patients for endovascular treatment $[10,21]$. Recently, a systematic review and meta-analysis in the patients receiving intravenous thrombolysis alone without endovascular treatment also found that good pre-treatment collaterals were associated with smaller infarct size at baseline, a lower rate of symptomatic intracranial hemorrhage and a higher rate of early neurological improvement [22].

We have found that three studies focusing on assessment of clinical and imaging parameters, including CTA collateral status, to identify predictive factors of mMCAi. Kim et al[4] retrospectively reviewed 64 acute stroke patients with MCA occlusion within 8 hours after the symptom onset. Both patients receiving endovascular treatment and only intravenous thrombolysis were included. Recanalization of occluded arteries occurred in 21 patients. Clinical, laboratory and imaging parameters were analyzed for malignant brain edema. Baseline NIHSS $>18$ and CTA collateral score $<2$, using the same grading system as in our study, were found to be independent predictors of malignant brain edema.

Jo et al[19] retrospectively analyzed larger population of 121 patients with MCA occlusion. Recanalization of occluded arteries was achieved in 57 patients. They found 4 factors that were independently associated with malignant brain edema; which were baseline NIHSS, baseline NECT ASPECTS, CTA collateral status and revascularization failure. The same CTA grading system as our study was used again. 
Flores et al[7] determined the impact of collateral circulation with development of mMACi, using multiphase CTA and The University of Calgary scoring system. Eighty-two patients with proximal MCA or ICA occlusion were prospectively included; 53 patients received endovascular treatment. Poor collateral circulation was found to be the only independent predictor of mMCAi.

In our study, we found a significant correlation between baseline NECT ASPECTS and CTA collateral status on development of mMCAi. After performing multivariate analysis, only baseline NECT ASPECTs was found to be an independent predictive factor for mMCAi. This discordant result may be due to the fact that we had a smaller number of patients and our patients may have lower baseline NECT ASPECTS compared to the mentioned studies.

We also found that patients with baseline ASPECTS $\leq 7$ significantly were more likely to develop mMCAi compared to patients with ASPECTS $>7$. This result is in agreement with the study by MacCallum et al [23].

Multiphase CTA was developed by the University of Calgary and used in ESCAPE trial as one of the criteria of patient selection for endovascular treatment $[17,18]$. Multiphase CTA provides information on the degree and extent of pial arterial filling in a time resolved manner. Compared to multiphase CTA, single phase CTA potentially mislabel the delayed pial arterial filling and may underdiagnose the true collateral status [17]. However, a number of studies using single phase CTA for assessment of collateral status in stroke patients also showed a significant impact on the clinical outcome in both endovascular treatment and intravenous thrombolysis patient groups $[21,22]$.

Our study has several potential limitations. First, our study is a retrospective study and may potentially have selection bias. Second, we did not have information about recanalization status that may affect the final infarct size. Finally, this study was performed at a single center and has a small sample size. 


\section{Conclusion}

In patients with acute stroke with proximal MCA or ICA occlusion received only intravenous thrombolysis, baseline NECT ASPECTS and collateral status evaluated by single phase CTA showed significant correlation with development of malignant MCA infarction. However, only baseline NECT ASPECTS $\leq 7$ was an independent predictor of malignant MCA infarction. CTA collateral status was not an independent predictive factor for this condition. 


\section{References}

1. Hacke W, Schwab S, Horn M, Spranger M, De Georgia M, von Kummer R. 'Malignant' middle cerebral artery territory infarction: clinical course and prognostic signs. Arch Neurol 1996;53:309-15.

2. Ropper AH, Shafran B. Brain edema after stroke. Clinical syndrome and intracranial pressure. Arch Neurol 1984;41:26-9.

3. Heiss WD. Malignant MCA infarction: pathophysiology and imaging for early diagnosis and management decisions. Cerebrovasc Dis 2016;41:1-7. doi:10.1159/000441627.

4. Kim H, Jin ST, Kim YW, Kim SR, Park IS, Jo KW. Predictors of malignant brain edema in middle cerebral artery infarction observed on CT angiography. J Clin Neurosci 2015; 22:554-60.

5. Subramaniam S, Hill MD. Massive cerebral infarction. Neurologist 2005;11:150-60.

6. Staykov D, Gupta R. Hemicraniectomy in malignant middle cerebral artery infarction. Stroke 2011;42:513-6. doi: 10.1161/STROKEAHA.110.605642.

7. Flores A, Rubiera M, Ribó M, Pagola J, Rodriguez-Luna D, Muchada M, et al. Poor collateral circulation assessed by multiphase computed tomographic angiography predicts malignant middle cerebral artery evolution after reperfusion therapies. Stroke 2015;46:3149-53. doi:10.1161/STROKEAHA. 115.010608 .

8. Liebeskind DS. Collateral circulation. Stroke 2003;34:2279-84. 
9. Nambiar V, Sohn SI, Almekhlafi MA, Chang HW, Mishra S, Qazi E, et al. CTA collateral status and response to recanalization in patients with acute ischemic stroke. AJNR Am J Neuroradiol 2014;35:884-90. doi: 10.3174/ajnr. A3817.

10. Menon BK, Qazi E, Nambiar V, Foster LD, Yeatts SD, Liebeskind D, et al. Differential effect of baseline computed tomographic angiography collaterals on clinical outcome in patients enrolled in the interventional management of stroke III trial. Stroke 2015;46:1239-44. doi: 10.1161/ STROKEAHA.115.009009.

11. Christoforidis GA, Karakasis C, Mohammad Y, Caragine LP, Yang M, Slivka AP. Predictors of hemorrhage following intra-arterial thrombolysis for acute ischemic stroke: the role of pial collateral formation. AJNR Am J Neuroradiol 2009;30:165-70. doi: 10.3174/ajnr.A1276.

12. Bang OY, Saver JL, Kim SJ, Kim GM, Chung CS, Ovbiagele B, et al. Collateral flow predicts response to endovascular therapy for acute ischemic stroke. Stroke 2011;42:693-9. doi: 10.1161/STROKEAHA.110.595256.

13. Liebeskind DS, Tomsick TA, Foster LD, Yeatts SD, Carrozzella J, Demchuk $\mathrm{AM}$, et al. Collaterals at angiography and outcomes in the Interventional Management of Stroke (IMS) III trial. Stroke 2014;45:759-64. doi: 10.1161/ STROKEAHA.113.004072.

14. Yeo LL, Paliwal P, Low AF, Tay EL, Gopinathan A, Nadarajah M, et al. How temporal evolution of intracranial collaterals in acute stroke affects clinical outcomes. Neurology 2016;86:434-41. doi:10.1212/WNL.0000000000002331.

15. Fanou EM, Knight J, Aviv RI, Hojjat SP, Symons SP, Zhang L, et al. Effect of collaterals on clinical presentation, baseline imaging, complications, and outcome in acute stroke. AJNR Am J Neuroradiol 2015;36:2285-91. doi: 10.3174/ajnr.A4453. 
16. Brunner F, Tomandl B, Hanken K, Hildebrandt H, Kastrup A. Impact of collateral circulation on early outcome and risk of hemorrhagic complications after systemic thrombolysis. Int J Stroke. 2014;9:992-8. doi: 10.1111/j.1747-4949.2012.00922.x.

17. Menon BK, d'Esterre CD, Qazi EM, Almekhlafi M, Hahn L, Demchuk AM, et al. Multiphase CT angiography: a new tool for the Imaging triage of pa tients with acute ischemic stroke. Radiology 2015;275:510-20. doi: 10.1148/ radiol.15142256.

18. Goyal M, Demchuk AM, Menon BK, Eesa M, Rempel JL, Thornton J, et al. Randomized assessment of rapid endovascular treatment of ischemic stroke. N Engl J Med 2015 ; 372:1019-30. doi: 10.1056/NEJMoa1414905.

19. Jo K, Bajgur SS, Kim H, Choi HA, Huh PW, Lee K. A simple prediction score system for malignant brain edema progression in large hemispheric infarction. PLoS One 2017; 12(2):e0171425. doi: 10.1371/journal. pone.0171425. eCollection 2017.

20. Berger C, Fiorelli M, Steiner T, Schäbitz WR, Bozzao L, Bluhmki E, et al. Hemorrhagic transformation of ischemic brain tissue: asymptomatic or symptomatic? Stroke 2001; 32:1330-5.

21. Leng X, Fang H, Leung TW, Mao C, Miao Z, Liu L, et al. Impact of collaterals on the efficacy and safety of endovascular treatment in acute ischaemic stroke: a systematic review and meta-analysis. J Neurol Neurosurg Psychiatry 2016;87:537-44. doi: 10.1136/jnnp-2015-310965.

22. Leng X, Lan L, Liu L, Leung TW, Wong KS. Good collateral circulation predicts favorable outcomes in intravenous thrombolysis: a systematic review and meta-analysis. Eur J Neurol 2016;23:1738-49. doi: 10.1111/ene.13111. 
THE ASEAN JOURNAL OF RADIOLOGY

23. MacCallum C, Churilov L, Mitchell P, Dowling R, Yan B. Low Alberta Stroke Program Early CT score (ASPECTS) associated with malignant middle cerebral artery infarction. Cerebrovasc Dis 2014;38:39-45. doi:10.1159/ 000363619 . 